\title{
Aberrancy in CNS Signals and other Factors Related to Altered Homeostasis, Obesity and Metabolic Syndrome
}

\author{
Vinod Nikhra* \\ Department of Medicine, Hindu Rao Hospital and NDMC Medical College, India
}

Submission: September 25, 2018; Published: November 20, 2018

*Corresponding author: Vinod Nikhra, Senior Chief Medical Officer and Consultant, Department of Medicine, Hindu Rao Hospital and NDMC Medical College, New Delhi, India

\begin{abstract}
Introduction: 'The Obese-Obese World': The obesity and metabolic syndrome (MetS) are a global epidemic of such magnitude that the today's health scenario can be summed up as the 'Obese-obese World'*. Obesity and MetS deteriorate quality of life and alter course of various chronic diseases, and on their own, are risk factors for diabetes, hypertension, cardiovascular disease and stroke, neurological degenerative diseases and cancers. Modern day lifestyle drives for excess calorie intake, comparatively reduced energy expenditure and storage of surplus energy in adipose tissue, an accentuated evolutionary need to fill body nutrients stores, leading to obesity, appended by pathophysiological alterations termed MetS.
\end{abstract}

Cns Regulation of Energy Intake: Specialised neurons in hypothalamus and brainstem primarily regulate energy homeostasis, food intake and body weight, and integrate multiple peripheral metabolic inputs, such as nutrients, gut-derived hormones, and adiposity-related signals. There are several neuropeptides involved, including melanin concentrating hormone (MCH) and the orexins. An abnormal alteration in ghrelin and leptin levels can lead to weight gain and Obesity. Increase in adipose tissue leads to overproduction of leptin and hypothalamus becoming resistant to leptin action. The reward circuitry involves interactions between several systems including opioids, endocannabinoids, serotonin and dopamine. The obese individuals appear to have abnormalities in dopaminergic activity, and an imbalance in the brain circuits promoting reward seeking and those governing cognitive control leads to an overriding stimulus to feeding, even in the absence of an energy deficit. Dorsal striatum is hyperactive in obese and may contribute aberrancy of satiety signals. The genetics involving various mutations contributes up to $70 \%$ towards a person's vulnerability to obesity.

Regulation of Energy Expenditure: Energy is consumed in processes of physical activity, basal metabolism, and adaptive thermogenesis, which are modulated by brain, especially hypothalamic melanocortin system. Brown adipose tissue (BAT) plays a major role in thermogenesis. Central regulation of BAT thermogenesis is dependent on sympathetic outflow to BAT. Norepinephrine released from sympathetic nerve terminals binds to $\beta 3$-adrenergic receptors on adipocytes in BAT to promote enhanced thermogenesis. In addition, many hormonal and nutrient signals, such as glucose, insulin, leptin and GLP-1 also influence sympathetic outflow to BAT.

Conclusion - Fallouts of Neurosignal Aberrancy: The obese subjects with BMI > 30 show atrophy in the frontal lobes, anterior cingulate gyrus, hippocampus, and thalamus. MetS affects various cognitive domains including executive functioning, processing speed, and overall intellectual functioning. There is impaired vascular reactivity, endothelial dysfunction, neuro-inflammation, oxidative stress and altered brain metabolism.

Keywords: Appetite regulation; Brown adipose tissue; Dopaminergic system; Endocannabinoids; Gastrointestinal peptides; Genetic mutations; Hypothalamic neurones; Neuroinflammation; Neurodegeneration

\section{Introduction}

The Obese-Obese World: The overweight and obesity are a global epidemic of such magnitude that the today's health scenario can be summed up as the 'Obese-obese World' [1]. Obesity and MetS deteriorate quality of life and alter course of various chronic diseases, and on their own, are risk factors for diabetes, hypertension, cardiovascular disease and stroke, neurological degenerative diseases and cancers. Modern day lifestyle drives for excess calorie intake, comparatively reduced energy expenditure and storage of surplus energy in adipose tissue, an accentuated evolutionary need to fill body nutrients stores, leading to obesity, appended by pathophysiological alterations termed MetS (Figure 1). The pandemic of nutrition-related non-communicable diseases (NR-NCD) is due to an increased consumption of processed foods, saturated and total fats, sugar, and high caloric beverages [2]. The current pandemic of NR-NCD has occurred in the setting a global reduction in physical activity [3]. According to the World Health Organization, insufficient physical activity was one of the 10 leading risk factors for mortality, contributing to some 3.2 million deaths per year, and 69.3 million disability-adjusted life years in 2010 [4]. 


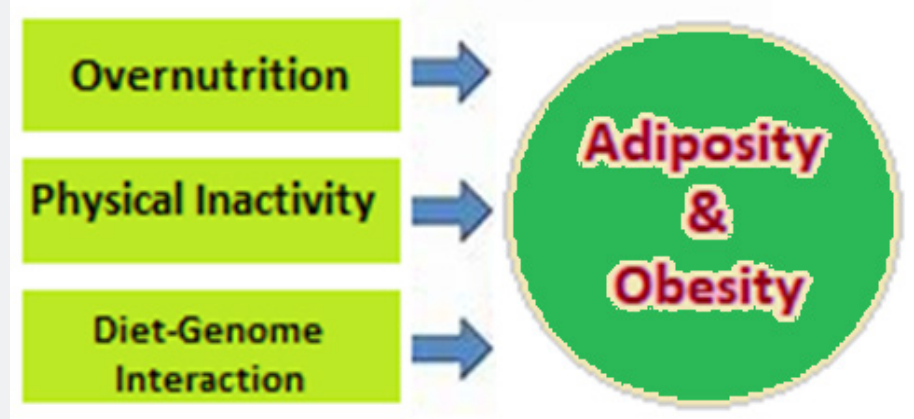

Figure 1: Gross Etiology of Pandemic of NR-NCD.

\section{CNS Regulation of Metabolic Homeostasis}

The specialised neurons in hypothalamus and brainstem primarily regulate energy homeostasis, food intake and body weight, and integrate multiple peripheral metabolic inputs, such as nutrients, gut-derived hormones and signals from adipose tissue [5]. There are several neuropeptides involved, including melanin concentrating hormone $(\mathrm{MCH})$ and the orexins [6]. The reward circuitry involves interactions between several systems including opioids, endocannabinoids, serotonin and dopamine. The obese individuals appear to have abnormalities in dopaminergic activity and an imbalance in the brain circuits promoting reward seeking and those governing cognitive control overriding stimulus to feeding [7]. The increase in adipose tissue leads to overproduction of leptin and hypothalamus becomes relatively resistant to its action. Dorsal striatum is hyperactive in obese and may contribute aberrancy of satiety signals. The genetics involving various mutations may contribute up to $70 \%$ towards a person's vulnerability to obesity.

\section{The Hypothalamus}

The hypothalamus is regulator of the autonomic nervous system (ANS), the circadian clock for behavioral and sleep-wake functions, the neural centres of the endocrine system, and the primary regulator of thirst and hunger. It communicates with other regulating centres in the central and peripheral nervous systems, including the prefrontal and insular cortices, the amygdala and other limbic structures, the midbrain, the pons, the medulla, and the vagus and glossopharyngeal nerves. There are projections to and from hypothalamus, brainstem, pons and cortical centers. The arcuate nucleus (AN) contains orexigenic neurons co-expressing agouti-related protein and neuropeptide Y (AgRP/NPY neurons) and anorexigenic neurons co-expressing pro-opiomelanocortin and cocaine and amphetamine-regulated transcripts (POMC/CART). AN plays a major role in regulation of food intake and calorie expenditure (Figure 2). Being located outside the blood-brain barrier, it is able to receive inputs from circulating nutrients and hormones. The AgRP/NPY expressing neurons enhance appetite and weight gain by $\gamma$-aminobutyric acid (GABA)-mediated tonic inhibition of POMC/CART neurons including the suppression of anorexigenic signals from neurons located in the parabrachial nucleus of the pons. The anorexigenic inputs come from glutaminergic signals originating in the nucleus tractus solitarius (NTS) in the medulla. Both insulin and leptin suppress appetite by inhibiting AgRP/NPY neurons. The orexigenic hormone ghrelin, produced by gastric mucosa, enhances food intake by activating AgRP/NPY neurons.

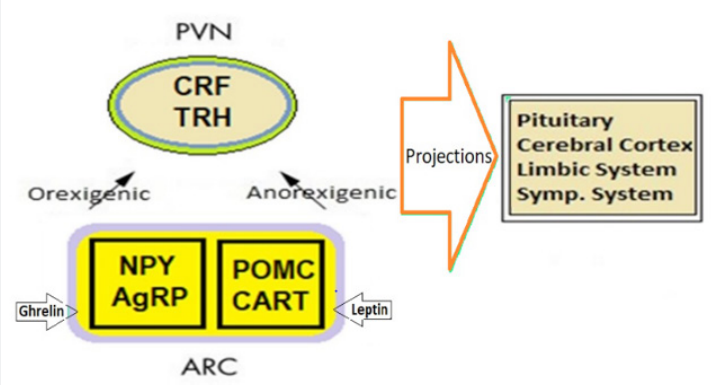

Figure 2: The CNS and GI System Regulating Energy Homeostasis.

The POMC/CART expressing neurons produce $\alpha$-melanocyte stimulating factor ( $\alpha$-MSF) which on binding to melanocortin-3 (MC3R) and melanocortin-4 (MC4R) receptors promotes energy expenditure and suppresses food intake [8]. These neurons are inhibited by NPY and activated by leptin, serotonin, brain-derived neurotrophic factor (BDNF), and glial-cell-derived growth and differentiation neurotrophic factor 15 (GDF15). Glucagon-like peptide-1 (GLP-1) expressing neurons in the medulla send anorexigenic signals to the paraventricular nucleus (PVN). Activation of the sympathetic nervous system by melanocortin receptors in the ventromedial (VM) nucleus enhances energy expenditure by increasing fatty acid utilization and thermogenesis in skeletal muscle and brown adipose tissue. 


\section{Area Postrema, NTS and Vagus}

The area postrema in brainstem receives and integrates multiple metabolic signals, including anorexigenic signals from leptin, cholecystokinin, amylin, GLP-1, pancreatic peptide (PP) and peptide YY (PYY), and orexigenic signals from ghrelin. This area projects to NTS, which receives input from vagal afferent fibers located in the gastric mucosa and intestinal wall and relays the signals to the parabrachial nucleus (PBN) in the pons, which send anorexigenic signals to the arcuate nucleus and central amygdala. GLP-1 expressing neurons in the NTS relay anorexigenic signals to corticotrophin-releasing hormone (CRH), nefstatin-1, and oxytocin-expressing neurons in the PVN. GLP-1 receptor binding restricts feeding by excitatory synaptic signals in paraventricular CRH neurons via a protein kinase A-dependent signalling cascade. The BDNF receptor, TrkB, is highly expressed in the area postrema, NTS and dorsal motor nucleus of the vagus, and mediates the anorexigenic effects of brainstem BDNF [9].

The vagal neurons expressing receptors for gut-derived hormones play a significant role in appetite regulation. GLP-1 binding induces an anorexigenic phenotype in afferent vagal neurons, an effect that is down-regulated by ghrelin. Further, the cholecystokinin-induced inhibition of food intake is dependent on signaling from vagal afferent neurons. Importantly, the vagal afferent neurons can change their phenotype and express either orexigenic or anorexigenic receptors depending on the availability of nutrients.

\section{Midbrain and Limbic System}

The taste aversions and taste preferences are mediated in part by nuclei located in the central amygdala. Smell sensation is also linked to appetite modulation [10,11]. However, food reward signals are mediated primarily by midbrain dopaminergic neurons projecting to the limbic system and the prefrontal cortex which modulates eating behaviour. Further, growth hormone secretagogue receptors (GHSRs) in dopaminergic neurons mediate ghrelin-induced feeding and food-reward behaviour.

\section{Altered CNS Control for Metabolic Homeostasis}

\section{The Hypothalamus}

In majority of obese persons, the ability of the hypothalamus to control energy balance is altered [12]. The aberrant functioning is present at multiple levels like integration of satiety signals and regulation of glucose and lipid metabolism. In addition, the over-nutrition and adiposity leading to inflammatory response alter the functioning of various hypothalamic nuclei [13]. The studies in rodents have documented that over-nutrition causes resistance to the anorexic and thermogenic effects of leptin due to reduced mRNA expression and transduction capabilities of the leptin receptors in AN [14]. The high fat diet reduces synapses in POMC neurons Horvath et al. (2010). The diet-induced obesity is associated with a failure of leptin to inhibit the orexigenic effects of AgRP neurons [15]. Humans with SNPs in the MC4R are morbidly obese, hyperphagic, and have IR and elevated leptin levels. The obese humans have reduced sensitivity to leptin's anorexigenic effects. Fasting ghrelin levels in children with the Prader-Willi syndrome are elevated and appear to play a role in their insatiable appetite. The BDNF levels are decreased in obese subjects with T2DM [16].

\section{Area Postrema, NTS, PBN and Vagus}

In rodents high-calorie diet impairs anorexigenic signals in the caudomedial nucleus of the NTS [17] and alters circadian clock genes [18]. Treatment with GDF15, binding to neurons in the NTS, the area postrema, and the arcuate nucleus, decreases adiposity and corrects metabolic function in diet-induced obese mice [19]. The administration of GLP-1 and GLP-1 agonists such as exendin- 4 and liraglutide reduce food intake and weight in obese rodents by inducing anorexigenic phenotype switching in afferent vagal neurons. In humans, plasma GLP-1 levels are low in obese individuals with normal or impaired glucose tolerance [20], and administration of native GLP-1 and GLP-1 agonists have been shown to reduce food intake and weight in obese humans [21]. In obese subjects, high intensity exercise increases GLP-1 levels and reduces hunger scores [22]. The therapeutic effects following bariatric surgery involve the vagal NTS and vaso-vagal pathways in restoring normal function to the pancreas - normalizing insulin secretion, reducing glucagon production; liver - recovering insulin sensitivity, reducing gluconeogenesis and free fatty acid release; and gastrointestinal tract - reducing ghrelin secretion, restoring normal responses to nutrients, peptides, hormones [23].

\section{Midbrain and Limbic System}

The physical inactivity in setting of obesity is associated with reductions in dopamine receptor signaling in the meso-accumbens dopamine system. Hedonic eating is the consumption of palatable foods beyond the need-based energy requirements and prevalent in overweight and obese individuals [24]. The remarkable success of gastric bypass surgeries in controlling overweight has been attributed, in part, to a reduction in hedonic eating. Studies in humans have shown that Roux-en-Y gastric bypass patients are less preoccupied with eating and begin to prefer low calorie over high calorie more palatable foods [25-27].

\section{Multivariate Factors and Aberrant CNS Regulation}

Energy balance and body weight are regulated by CNS, which senses metabolic status from various humoral and neural signals and controls energy intake [28]. In the hypothalamus, stimulation of leptin- and ghrelin-responsive pathways, including the central melanocortin system, contributes to the maintenance of nearly stable adipose tissue amount and body weight [29]. The CNS melanocortin (MC) system is implicated as a mediator of the central effects of leptin, and reduced activity of the CNS-MC system promotes obesity in both rodents and humans. The CNS$\mathrm{MC}$ receptor activation normally boosts metabolic rate. In addition to $\alpha-\mathrm{MSH}$, a number of other hypothalamic neuropeptides are known to be involved in the regulation of energy balance. These include corticotropin-releasing hormone ( $\mathrm{CRH}$ ), neuropeptide $\mathrm{Y}$ (NPY), and cocaine-amphetamine-related transcript (CART). The 
activity of these neuropeptide systems is controlled by leptin, and possibly by melanocortins as well [30].

The dysregulation of the pathways is a marker of changes in energy balance [31]. Ghrelin has appetite-inducing activities and acts as an afferent signal to the hypothalamus. Leptin acts as an afferent signal from adipose tissue to the hypothalamus, as a part of negative feedback loop regulating the size of energy stores and energy balance. Ghrelin is negatively correlated with weight and obese subjects have lower ghrelin levels than lean subjects, consistent with a compensatory rather than causal role for ghrelin in obesity. On the contrary, circulating leptin levels correlate with proportion to adiposity, suggesting that obesity is associated with insensitivity to leptin, i.e., leptin resistance. Apparently, ghrelin and leptin operate as metabolic switch through hypothalamic/ pituitary axis [32]. The appetite, energy expenditure, and basal metabolic rate are linked [33,34].

The CNS significantly regulates adipose tissue mass by acting on the metabolic pathways and on the adipose plasticity. Innervation by autonomic nerves modulates glucose and fat metabolism in adipose tissue and adipose tissue functions at the cellular and molecular level like modulation of lipolysis/ lipogenesis, local insulin sensitivity of glucose and fatty acid uptake, the expression levels of several adipokines in adipose tissue, and the adipose tissue amount and cell-size. In general, the sympathetic nervous system is related to catabolism and the parasympathetic system to anabolism [35].

The increased release of pro-inflammatory cytokines, including TNF- $\alpha$, is a key feature of the pathophysiology of metabolic disorders. Adiposity induces an inflammatory response in peripheral metabolic tissues. The metabolic inflammation or meta-flammation, causes metabolic defects that underlie T2DM and obesity. TNF- $\alpha$ is overexpressed in adipose tissue of obese individuals and the elevated TNF- $\alpha$ levels cause peripheral IR. In T2DM, elevated TNF- $\alpha$ levels trigger serine phosphorylation of IRS- 1 by stress kinases, which interferes with its ability to engage in insulin receptor signaling and blocks the intracellular actions of insulin. The blockade of TNF- $\alpha$ in obese mouse models results in improved insulin sensitivity and glucose homeostasis. In the brain, TNF- $\alpha$ is secreted mainly by microglial cells in response to trauma, infection or abnormal accumulation of protein aggregates [36].

Lipids are structural elements of membranes or act as signaling molecules regulating metabolic homeostasis through many mechanisms. With increasing adiposity, lipid influx can exceed the adipose tissue storage capacity, resulting in lipid accumulation at ectopic sites such as liver and muscle. The accumulation of lipids or generation of signaling intermediates can interfere with immune regulation, causing a chronic low-grade metabolic inflammation, termed meta-flammation, which is the hallmark of lipotoxicity in metabolic diseases such as obesity and diabetes, and occurs in several organs including adipose tissue, liver, muscle, brain and gut, and influences several immuno-metabolic pathways [37].

i. The lipotoxicity and accumulation of lipid cause deterioration of metabolic regulation by converging on inflammatory and stress pathways.

ii. Lipotoxicity can also lead to ROS production from mitochondria and inflammasome activation. The synthesis of DAGs and ceramides.

iii. DAGs activate stress kinases, PKCs, and the NFkB pathway, and ceramides activate JNK signaling. Both DAGs and ceramides cause insulin resistance via inhibition of IRS1 and AKT, respectively.

iv. Activation of TLR4 signaling, which leads to activation of inflammasomes and induction of inflammatory gene transcription factors interferon regulatory factor (IRF), NFKB, and AP-1.

V. Accumulation of oxidized cholesterol or cholesterol crystals also leads to induction of TLR4, PKR, and stress kinase (JNK and p38) signaling or inflammasome activation and proinflammatory gene expression, which are central players in atherosclerosis progression.

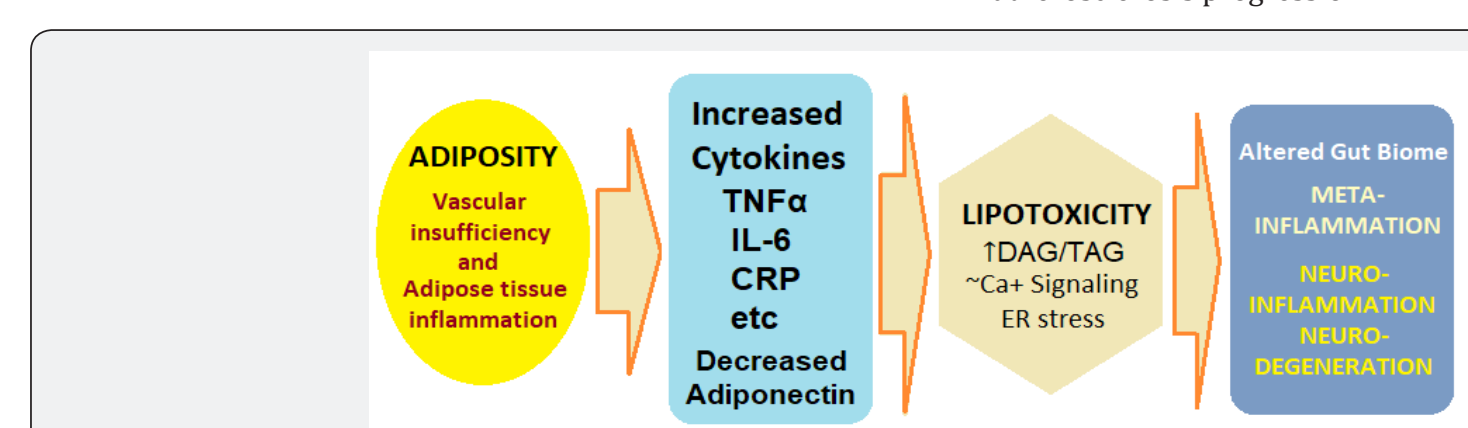

Figure 3: The Lipotoxicity and its Fallouts.

The mechanisms underlying the harmful effects of excess lipid flux are related in part to the impact of lipids on the biophysical properties of cellular organelles. The lipid synthesis is dysregulated in the ER, leading to changes in phospholipid composition of the ER membrane. These changes cause disruption of calcium signalling, prolonged ER stress, and decreased translation of ERassociated proteins (Figure 3). Whereas, saturated fatty acids and cholesterol loading increase ER stress and associated cell death. ER stress responses also intersect with inflammatory pathways via activation of numerous inflammatory kinases, such as JNK, 
protein kinase R (PKR), and IKK, and activation of inflammatory mediators and the inflammasome. Beyond the alteration of organelle function, lipotoxicity influences metaflammation and hormone action via direct effects on intracellular signalling pathways. Finally, lipids can influence cell fate and function by engaging receptors on the cell surface or stress kinases within the cytoplasm.

Lipotoxicity plays an important role in islet dysfunction. The chronic elevation of lipids leads to $\beta$-cell failure together with inflammatory etiology. T2DM patients have increased IL-1 $\beta$ expression and macrophage recruitment in the islets. Also, the $\beta$-cell failure in T2DM may have an inflammatory component that is compounded by lipotoxicity. In both humans and preclinical models, obesity-induced inflammatory changes are evident in the brain, and hypothalamic ER stress contributes to defective insulin and leptin action.

i. Skeletal muscle: High levels of circulating fatty acids and triglycerides are associated with muscle insulin resistance. In addition, adipocytes can accumulate in the muscle tissue. The lipid-mediated changes during IR in muscle converge with immune pathways and regulate inflammatory signaling.

ii. The heart has a robust capacity to utilize fatty acids for metabolic and functional demands. However, a prolonged increase in circulating fatty acids and triglycerides and accumulation of pericardial adipose tissue can trigger inflammatory signaling in the heart and cause cardiac dysfunction.

iii. The ingestion of high-fat diet leads to changes in the gut microbiome, and a contributor to development of metabolic disease. Gut-derived lipid signals such as N-acyl-phosphatidylethanolamines (NAPEs) produced on high fat intake have impact on immune-metabolic outcomes and the alterations in gut microbiota influence neuroinflammation [38].

As metaflammation is the hallmark of chronic metabolic disease, immunoregulatory or anti-inflammatory therapies can help reduce lipid-induced inflammation and cellular dysfunction. The $\omega$ - 3 fatty acids have also been shown to inhibit metabolic inflammation and alleviate insulin resistance. Additional beneficial roles are attributed to $\omega-3$ fatty acids through their metabolism into resolving and protectins. Because lipotoxicity impairs ER function and leads to a prolonged unfolded protein response, which can engage stress and inflammatory pathways. The use of agents that alleviate ER stress, such as tauro-ursodeoxy-cholic acid (TUDCA) and 4-phenylbutyric acid, has been tested in metabolic contexts with beneficial results on liver and CNS function. TUDCA treatment in experimental models of acute pancreatitis and ischemia reperfusion in liver also demonstrated decreased JNK activity along with attenuated inflammatory responses. Interestingly, TUDCA treatment in humans also improved liver and muscle insulin resistance warranting further clinical studies.

\section{Neuroinflammation and Neurodegeneration}

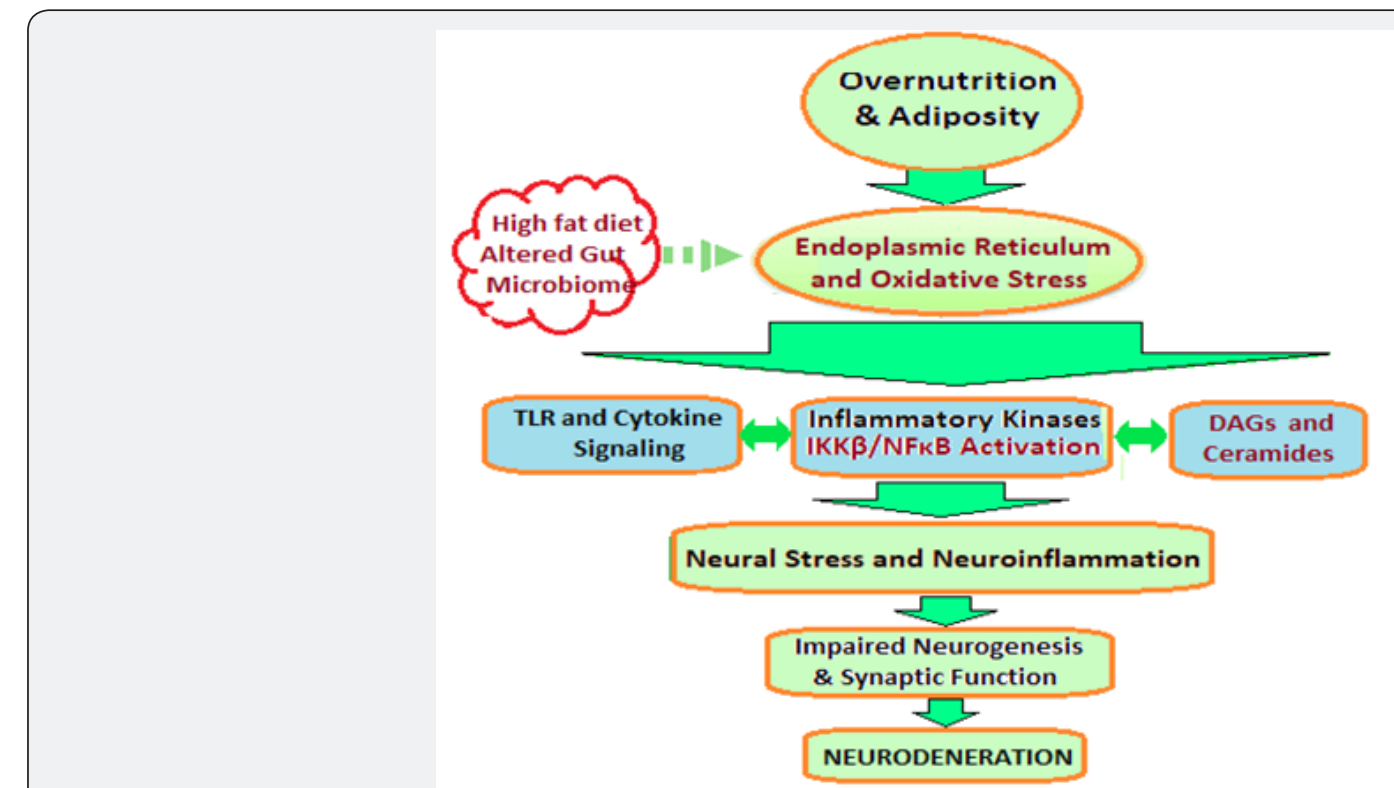

Figure 4: Multivariate Factors and Neuro-Inflammation-Degeneration.

Obesity directly affects the glucose and energy metabolism of the brain cells and through increased secretion of pro-inflammatory agents like TNF- $\alpha$, IL-1, IL-6 induces neuroinflammation primarily in the hypothalamic area of the brain. The overall effect is impairment of neuronal function and internal molecular damage, which results in abnormal protein deposits intracellularly or extracellularly or both, leading to neurodegeneration (Figure 4). Obesity and neurodegenerative diseases (NDDs) are linked by alterations in molecular pathways such as $\mathrm{P} 13 \mathrm{~K} /$ Akt signaling pathway and IKK $\beta / \mathrm{NF}-\mathrm{\kappa B}$ pathway, which change genes expression profiles and activate or deactivate molecular mediators, heralding the drift away from normal cellular functioning. Concomitants with these alterations are oxidative damage to cellular components and increased secretion of pro- 
inflammatory factors such as TNF $\alpha$, cytokines and interleukins [39].

\section{Impaired Glucose Homeostasis and CNS Physiology}

Impaired glucose tolerance precedes T2DM which in turn leads to various neurodegenerative disorders. As compared to general population, the increased risk of dementia is $50 \%-150 \%$ in those with T2DM. Insulin holds important neurotrophic properties in the brain. It is transported to the central nervous system through the blood-brain barrier by a transport mechanism mediated by insulin receptors. These receptors are chiefly localized in hippocampus, entorhinal cortex and frontal which functions in learning, memory and cognition. Visceral adiposity is a major cause of insulin resistance. Visceral fat tissues due to their high metabolic rate act as endocrine organs that secretes adipokines (e.g. leptin) and cytokines (e.g. TNF- $\alpha$, IL-6, heparinbinding epidermal growth factor). Activation of proinflammatory pathways and secretion of cytokines leads to insulin resistance. Insulin resistance is the common pathophysiologic characteristic of obesity and glucose intolerance affecting the membrane cation transport. The insulin deficiency and resistance trigger neuronal death and neurodegeneration due to the withdrawal of trophic factor, energy metabolism deficits and inhibition of insulinresponsive gene expression.

Brain cells are unable to synthesize or store glucose; therefore, it has to be transported across the blood-brain-barrier. This is done by Glucose Transporters like GLUT-1, GLUT-3 and GLUT-4. In condition of glucose dysmetabolism, advanced glycation end products (AGEs), start accumulating within the cells. AGEs glycated $A \beta$ which make these peptides more prone to aggregation, also AGEs play a role in hyperphosphorylation of tau, and in formation of senile plaques, tau hyperphosphorylation and subsequently, neurofibrillary tangles, the hallmark for AD.

\section{Initiation and Sustenance of Neuroinflammation}

Neuroinflammation is activation of innate immune response of brain for protection of CNS against infections, injuries and disease, and comprises of a complex series of reactions consisting of cellular and molecular changes, activation of peripheral immune response, initiation of intracellular signalling pathway, release of inflammatory mediators leading to neuronal dysfunction and loss. Neuroinflammation leads to neurodegeneration by activation of IKK $\beta / N F-\kappa B$ pathway, dysfunction of BBB and accumulation of macrophages, astrocytes and microglia. Obesity facilitates systemic inflammation as the hypertrophied adipocytes and immune cells of adipose tissue lead to increased circulating levels of proinflammatory cytokines like TNF- $\alpha$, IL- 6 , IL-1 $\beta$ etc. IL- 6 and IL-1 $\beta$ have been shown to damage neuronal circuits involved in cognition and memory. Thus, neuroinflammation can, thus, be considered a consequence of obesity leading to neurodegeneration.

Over 50 adipokines are produced by adipocytes, out of which leptin, autotaxin and adiponectin play predominant role in neurodegeneration. Leptin, a $16 \mathrm{KDa}$ protein, is translated from obese (ob) gene, and regulates appetite by primarily acting on hypothalamic region. Leptin receptors are also expressed in extra hypothalamic regions like amygdala, brain stem and cerebellum. Leptin is neuroprotectant against oxidative stress and cytotoxicity. Leptin has protective effects against 6-OHDA (6-hydroxydopamine) toxicity in dopaminergic neurons and preserves the functioning of the dopamine system. The reduced leptin levels with obesity, increase in the risk of PD. The leptin signaling is significantly decreased in the arcuate nucleus in obese individuals [40] and has been negatively correlated with AD pathology. Obesity has also been associated with the accelerated aging process.

Hypothalamus constitutes the major CNS part affected by neuroinflammation. IKK $\beta / N F-\kappa b$ has been documented to trigger hypothalamic inflammation which in turn leads to glucose intolerance, insulin resistance and impaired insulin secretion. Activation of IKK $\beta / \mathrm{NF}-\mathrm{kb}$ pathway also affects the differentiation of neuronal stem cells and impairs their survival resulting in neurodegeneration.

\section{Alterations in Signaling Pathways leading to Neurode- generation}

Insulin and Insulin Growth Factor 1 (IGF-1) are important in brain glucose homeostasis and cell survival, and act through the P13K-Akt signaling pathway. In normal insulin conditions, insulin receptors (IR)/IGF-1Rs are activated in response to oxidative stress, whereas glycogen synthasekinase-3 $\beta$ (GSK$3 \beta$ ) is inhibited. This is accompanied by increased production of 4-hydroxynonenal (4-HNE) for oxidative protection of neuronal lipids and proteins. However, the impaired insulin/IR and IGF-1/ IGF-1R signaling result due to altered insulin and/or IGF-1 levels in obesity and T2DM.

The inflammatory response is mediated by the activation of $\mathrm{IKKb} / \mathrm{NF}-\kappa \mathrm{B}$ pathway. Hypothalamic inflammation induced by $\mathrm{IKKb} / \mathrm{NF}-\mathrm{kB}$ causes glucose intolerance and insulin resistance [41]. The IKK $\beta$ pathway degrades IkB protein and liberates NF$\kappa B$ which localises to the nucleus and activates transcription of inflammatory proteins. The Toll-like receptors (TLRs) and cytokine receptors have been shown to mediate neuroinflammation by activation of IKKb/NF- $\mathrm{BB}$ pathway through ER stress and autophagy defects. These various pathway alterations in obesity affect the normal brain functioning, lead to damaging effects on the brain cells through oxidative stress, ER stress and mitochondrial dysfunction, and cause cellular dysfunctions and gradual neuronal loss [42].

\section{References}

1. Nikhra Dr Vinod (2005) Chapter One: The Obese-obese World, in the book. The Anti-obesity Guide. Sahni Publications, Delhi, India.

2. Demmier KM, Klasen S, Nzuma JM, Qaim M (2017) Supermarket purchase contributes to nutrition-related non-communicable diseases in urban Kenya. PLoS ONE 12: e0185148.

3. WHO (2015) Global Health Estimates. 2015. Deaths by Cause, Age, Sex, by Country and by Region, 2000-2015. World Health Organization, Switzerland. 
4. John KS (2018) Exercise, Obesity and CNS Control of Metabolic Homeostasis: A Review. Front Physiol 9: 574.

5. Wu Q, Clark MS, Palmiter RD (2012) Deciphering a neuronal circuit that mediates appetite. Nature 483(7391): 594-598.

6. Williams KW, Scott MM, Elmquist JK (2011) Modulation of the central melanocortin system by leptin, insulin, and serotonin: coordinated actions in a dispersed neuronal network. Eur J Pharmacol 660(1): $2-12$.

7. Zhu X, Ottenheimer D, DiLeone RJ (2016) Activity of D1/2 receptor expressing neurons in the nucleus accumbens regulates running, locomotion, and food intake. Front. Behav Neurosci 10: 66 .

8. Gavini CK, Jones WC, Novak CM (2016) Ventromedial hypothalamic melanocortin receptor activation: regulation of activity energy expenditure and skeletal muscle thermogenesis. J Physiol 594(18) 5285-5301.

9. Spaeth AM, Kanoski SE, Hayes MR, Grill HJ (2012) TrkB receptor signaling in the nucleus tractus solitarius mediates food intakesuppressive effects of hindbrain BDNF and leptin. Am J Physiol Endocrinol Metab 302(10): E1252-1260.

10. Garrison JL, Knight ZA (2017) Linking smell to metabolism and aging. Science 358(6364): 718-719.

11. Riera CE, Tsaousidou E, Halloran J, Follett P, Hahn O, et al. (2017) The sense of smell impacts metabolic health and obesity. Cell Metab 26(1): 198-211.

12. Williams LM (2012) Hypothalamic dysfunction in obesity. Proc Nutrit Soc 71(4): 521-533.

13. McNay DEG, Briançon N, Kokoeva MV, Maratos-Flier E, Flier JS (2012) Remodeling of the arcuate nucleus energy-balance circuit is inhibited in obese mice. J Clin Invest 122(1): 142-152.

14. Patterson CM, Bouret SG, Dunn-Meynell AA, Levin BE (2009) Three weeks of postweaning exercise in DIO rats produces prolonged increases in central leptin sensitivity and signaling. Am J Physiol Regul Integr Comp Physiol 296(3): R537-548.

15. Baver SB, Hope K, Guyot S, Bjørbaek C, Kaczorowski C, et al. (2014) Leptin modulates the intrinsic excitability of AgRP/NPY neurons in the arcuate nucleus of the hypothalamus. J Neurosci 34(16): 5486-5496.

16. Li B, Lang N, Cheng ZF (2016) Serum levels of brain-derived neurotrophic factor are associated with diabetes risk, complications, and obesity: a cohort study from Chinese patients with type 2 diabetes. Mol Neurobiol 53(8): 5492-5499.

17. Cavanaugh AR, Schwartz GJ, Blouet C (2015) High-fat feeding impairs nutrient sensing and gut brain integration in the caudomedial nucleus of the solitary tract in mice. PLoS One 10(3): e0118888.

18. Kaneko K, Yamada T, Tsukita S, Takahashi K, Ishigaki Y, et al. (2009) Obesity alters circadian expressions of molecular clock genes in the brainstem. Brain Res 1263: 58-68.

19. Tsai VW, Zhang HP, Manandhar R, Lee-Ng KKM, Lebhar H, et al. (2017) Treatment with the TGF-b superfamily cytokine MIC-1/GDF15 reduces the adiposity and corrects the metabolic dysfunction of mice with dietinduced obesity. Int J Obes 42(3): 561-571.

20. Hussein MS, Abushady MM, Refaat S, Ibrahim R (2014) Plasma levels of glucagon-like peptide 1 in obese Egyptians with normal and impaired glucose tolerance. Arch Med Res 45(1): 58-62.

21. Näslund E, King N, Mansten S, Adner N, Holst JJ, et al. (2004) Prandial subcutaneous injections of glucagon-like peptide- 1 cause weight loss in obese human subjects. Br J Nutr 91(3): 439-446.

22. Ueda SY, Nakahara H, Miyamoto T (2013) Effects of exercise on glucagon-like peptide-1 (GLP-1). J Phys Fitness Sports Med 2(2):221224
23. Blasi C (2016) The role of the vagal nucleus tractus solitarius in the therapeutic effects of obesity surgery and other interventional therapies on type 2 diabetes. Obes Surg 26(12): 3045-3057.

24. Clemmensen C, Müller TD, Woods SC, Berthoud HR, Seeley RJ, et al. (2017) Gut-brain cross-talk in metabolic control. Cell 168(5): 758-774.

25. Ernest B, Thurnheer M, Wilms B, Schultes B (2009) Differential changes in dietary habits after gastric bypass versus gastric banding operations. Obes Surg 19(3): 274-280.

26. Schultes B, Ernst B, Wilms B, Thurnheer M, Hallschmid M (2010) Hedonic hunger is increased in severely obese patients and is reduced after gastric bypass surgery. Am J Clin Nutr 92(2): 277-283.

27. Ullrich J, Ernst B, Wilms B, Thurnheer M, Schultes B (2013) Roux-en Y gastric bypass surgery reduces hedonic hunger and improves dietary habits in severely obese subjects. Obes Surg 23(1): 50-55.

28. Myers MG, Olson DP (2012) Central nervous system control of metabolism. Nature 491(7424): 357-363.

29. Mason BL, Wang Q Zigman JM (2014) The central nervous system sites mediating the orexigenic actions of ghrelin. Annu Rev Physiol 76: 519533.

30. Adage T, Scheurink AJW, de Boer SF, de Vries K, Konsman JP, et al (2001) Hypothalamic, Metabolic, and Behavioral Responses to Pharmacological Inhibition of CNS Melanocortin Signaling in Rats. J Neurosci 21(10): 3639-3645.

31. Ronveaux CC, Tomé D, Raybould HE (2015) Glucagon-like peptide 1 interacts with ghrelin and leptin to regulate glucose metabolism and food intake through vagal afferent neuron signaling. J Nutr 145(4): 672-680.

32. Popovic V, Duntas LH (2005) Brain somatic cross-talk: ghrelin, leptin and ultimate challengers of obesity. Nutr Neurosci 8(1): 1-5.

33. Krawczewski Carhuatanta KA, Demuro G, Tschöp MH, Pfluger PT, Benoit SC, et al. (2011) Voluntary exercise improves high-fat dietinduced leptin resistance independent of adiposity. Endocrinology 152(7): 2655-2664.

34. Martins C, Morgan LM, Bloom SR, Robertson MD (2007) Effects of exercise on gut peptides, energy intake and appetite. J Endocrinol 193(2): 251-258.

35. Romijn JA, Fliers E (2005) Sympathetic and parasympathetic innervation of adipose tissue: metabolic implications. Curr Opin Clin Nutr Metab Care 8(4): 440-444.

36. Bartness TJ, Kay Song C, Shi H, Bowers RR, Foster MT (2005) Brainadipose tissue cross talk. Proc Nutr Soc 64(1): 53-64.

37. Ertunc ME, Hotamisligil GS (2016) Lipid signaling and lipotoxicity in metaflammation: indications for metabolic disease pathogenesis and treatment. The Journal of Lipid Research 57(12): 2099-2114.

38. Nho K, Paisley AK, Dehkordi SM, Arnold M, Risacher SL, et al. (2018) Altered Bile Acid Profile in Mild Cognitive Impairment and Alzheimer's Disease: Relationship to Neuroimaging and CSF Biomarkers. Alzheimer's Dement S1552-5260(18)33521-33530.

39. Gupta R, Sawhney P, Ambasta R, Pravir Kumar (2015) Obesity and neurodegeneration. Adv Obes Weight Manag Control. 2015;2(5):96-101.

40. de Git KC, Adan RA (2015) Leptin resistance in diet-induced obesity: the role of hypothalamic inflammation. Obes Rev 16(3): 207-224.

41. Arkan MC, Hevener AL, Greten FR, Maeda S, Li ZW, et al. (2005) INK- $\beta$ links inflammation to obesity-induced insulin resistance. Nat Med 11(2): 191-198.

42. Dongsheng Cai (2012) Neuroinflammation and Neurodegeneration in Overnutrition-induced Diseases. Trends Endocrinol Metab 24(1): 4047. 
This work is licensed under Creative Commons Attribution 4.0 License

DOI: 10.19080/CTBEB.2018.17.555960
Your next submission with Juniper Publishers will reach you the below assets

- Quality Editorial service

- Swift Peer Review

- Reprints availability

- E-prints Service

- Manuscript Podcast for convenient understanding

- Global attainment for your research

- Manuscript accessibility in different formats

(Pdf, E-pub, Full Text, Audio)

- Unceasing customer service

Track the below URL for one-step submission https://juniperpublishers.com/online-submission.php 